\title{
Die Komplikationenliste der Schweizerischen Gesellschaft für Innere Medizin
}

M. Stäubli, J. Suter

\section{Zusammenfassung}

Die Chefärztevereinigung der Schweizerischen Gesellschaft für Innere Medizin führt eine prospektive Erfassung der Komplikationen medizinischer Interventionen in Spitälern durch. Es ist das Ziel, durch die Weiter- und Fortbildung sowie durch organisatorische Massnahmen Häufigkeit und Schweregrad von Komplikationen zu vermindern und damit die Patientensicherheit zu verbessern. Mittels eines Mehrfachauswahlverfahrens werden bei jedem hospitalisierten Patienten Art und Anzahl der Interventionen sowie Art und Anzahl der Komplikationen erfasst. Alle Daten werden zentral bearbeitet und allen Kliniken zweimal pro Jahr anonymisiert und mit Expertenkommentaren zur Verfügung gestellt, die in Empfehlungen für die Optimierung von diagnostischen und therapeutischen Verfahren münden. Die seit sechs Jahren gewonnenen Erkenntnisse belegen die gute Durchführbarkeit des Programms, in welches bis Ende 2003 138000 Patienten einbezogen werden konnten.

\section{Einleitung}

Die Chefärztevereinigung der Schweizerischen Gesellschaft für Innere Medizin stellt den Spitälern seit 1998 das Programm «Komplikationenliste» zur Verfügung, das zum Ziel hat, Komplikationen zu vermindern [1-3]. Es dient der Analyse von Komplikationen der medizinischen Eingriffe. Das Programm ist in den Rahmen der Konzepte zur Verbesserung der Patientensicherheit einzureihen; die Patientensicherheit ist der wesentlichste Teil dessen, was man unter Qualitätssicherung in der Medizin verstehen muss.

\section{Spitalübergreifendes Programm}

Mit einem anonymisierten Rapportsystem werden die Komplikationen der diagnostischen und therapeutischen Eingriffe bei stationären Patienten erfasst. Durch kasuistische und statistische Analyse der Komplikationen werden Empfehlungen zur Prävention formuliert. Das Pro- gramm ist spital- und kantonsübergreifend: Durch die Beteiligung einer ganzen Anzahl von Kliniken werden auch Komplikationen erfasst, die im einzelnen Spital z. B. nur einmal oder nie pro Chefarztgeneration vorkommen, die aber, wenn man das Problem landesweit betrachtet, quantitativ plötzlich relevant werden. Spitäler, denen eine bestimmte Komplikation bzw. ein entsprechender Fehler noch nie passiert ist, erhalten mit dem Programm die nötige Information, um trotzdem Präventionsmassnahmen zu treffen. Voraussetzung zur Prävention ist, dass man anerkennt, dass eine bestimmte Komplikation auch im eigenen Spital vorkommen könnte, wenn sie in einem anderen möglich war.

Ein Gedankenexperiment kann das verdeutlichen: Schliesst man alle internmedizinischen Betten der Schweiz in einer einzigen «Klinik Schweiz» zusammen, ergibt das eine Institution mit etwa 10000 Betten. Diese wurden im Jahr 2002 von 312000 stationären Patienten benützt [4]. Das entspricht umgerechnet 170 Kliniken mit je etwa 1800 Patienten. Eine Komplikation, die in einem solchen Regionalspital einmal pro Jahr vorkommt, käme in der «Klinik Schweiz» $170 m a l$ pro Jahr vor. Eine Komplikation, die einmal pro acht Jahre im einzelnen Spital vorkommt, betrifft die «Klinik Schweiz» immerhin 21mal (Abb. 1).

Eine solche Komplikation wäre für die Leitung dieser Klinik wohl dringender Anlass zu strikten Präventionsmassnahmen, falls möglich; im kleinen Spital aber, wo man ihr nur alle acht Jahre begegnet und die Belegschaft inzwischen zum Teil gewechselt hat, wird man sie möglicherweise wieder als neu und einmalig zur Kenntnis nehmen und deshalb weniger alarmiert und zur Schaffung von Präventionsrichtlinien gedrängt sein. Eines der wichtigsten Ziele unseres Programmes ist es deshalb, diese Erfahrungslücken durch die spitalübergreifende Erhebung zu verkleinern, indem wir die Probleme der «Klinik Schweiz» den einzelnen Spitälern bekanntmachen, damit die mögliche Prävention überall stattfinden kann und wir dadurch $\mathrm{zu}$ einem «Qualitätsstandard Schweiz» gelangen. Dem Toten nützt es nichts, dass die tödliche Komplikation nur selten vorkommt. 
Abbildung 1

Hochrechnung der Komplikationshäufigkeit für den Bereich Innere Medizin mit 170 Kliniken in der Schweiz.

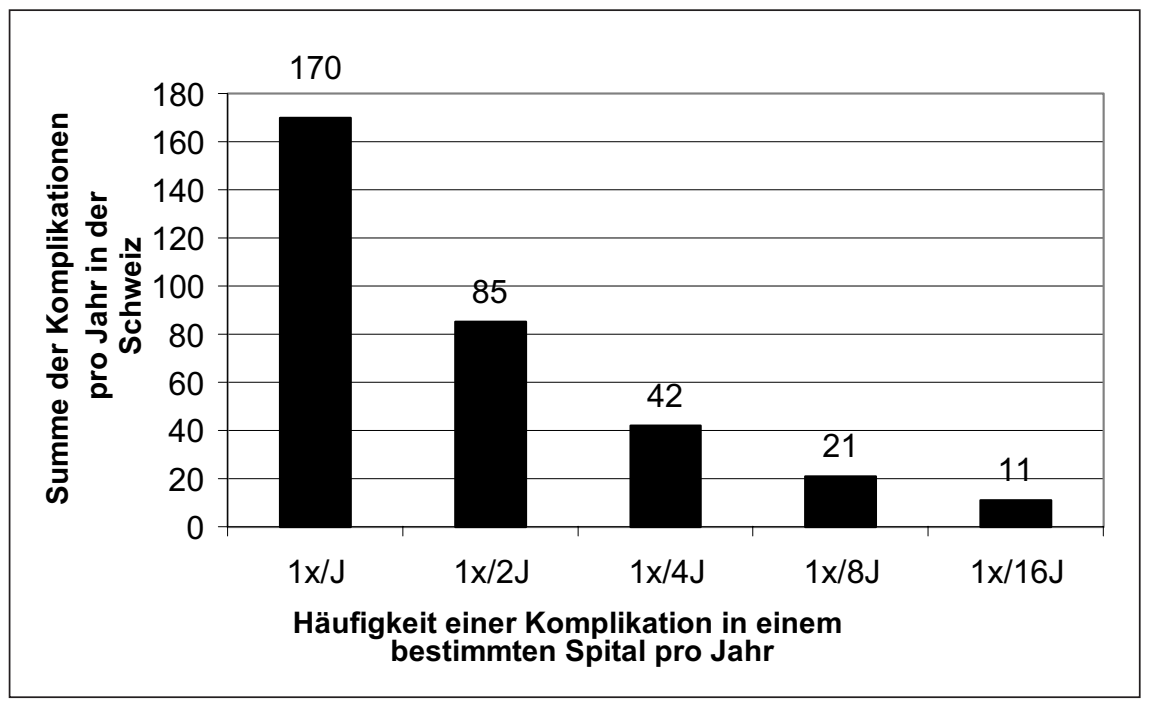

\section{Definition}

Als Komplikationen definieren wir unerwünschte Folgen medizinischer Interventionen, die im kausalen Zusammenhang mit dem Aufenthalt in einer internistischen Spitalabteilung auftreten. Es werden sowohl Komplikationen registriert, die auf Fehlern beruhen, als auch solche, die ohne fehlerhafte Handlung entstehen. Als Komplikationen werden wegen potentiell schwerwiegenden Folgen auch Vorkommnisse gewertet, aus denen keine tatsächlichen Nachteile für den Patienten resultieren: Verwechslungen, falsche Indikationen, falsche Dosierungen, Nichtdurchführung von notwendigen Massnahmen und ähnliches. Die Registrierung aller Interventionen und aller Komplikationen erlaubt die Angabe der relativen Komplikationshäufigkeit für jede Interventionsart.

\section{Das DuPont-Konzept im industriellen Bereich als Leitidee}

Unser prinzipielles Vorgehen lässt sich gut mit einem Unfallmeldesystem vergleichen, welches von der Firma DuPont seit über 100 Jahren entwickelt und in industriellen Betrieben angewendet wird.

Durch systematische Erfassung der Unfälle, auch der Bagatellen, sowie der unsicheren Handlungen und durch Elimination solcher Ursachen war das Unternehmen in der Lage, die Zahl der schweren bis tödlichen Unfälle im proportionalen Verhältnis zur Reduktion der Bagatellereignisse zu vermindern. Die Proportionalität zwischen der Anzahl unsicherer Handlungen und Bedingungen und den leichteren und schwereren Unfällen sowie den Unfällen mit Todesfolge kann durch eine Pyramide dargestellt werden (Abb. 2).

Wenn an der Basis der Pyramide die Anzahl unsicherer Handlungen und Bedingungen abnimmt, reduzieren sich die Unfallzahlen inklusive jener mit tödlichem Ausgang entsprechend. So ist es der Firma DuPont gelungen, die Unfallzahlen auf 1\% des Branchendurchschnittes zu senken. Wesentliche Erkenntnisse aus diesem Programm sind folgende:

- Unfälle sind keine Schicksalsschläge;

- Unfälle sind meistens die Folge von unsicheren Handlungen und unsicheren Bedingungen;

- es besteht ein signifikanter Zusammenhang zwischen der Anzahl unsicherer Handlungen oder Bedingungen und der Anzahl leichter bis schwerer Unfälle inklusive jener mit Todesfolge;

- durch Reduktion aller (auch der scheinbar unbedeutenden) unsicheren Handlungen und Bedingungen kann die Zahl leichter und schwerer Unfälle reduziert werden.

\section{Konzept Komplikationenliste}

Das analoge Konzept der Komplikationenliste besteht in der systematischen Erfassung aller unsicheren Handlungen und Bedingungen sowie Komplikationen bei medizinischen Handlungen, unabhängig vom Schweregrad, zwecks laufender Reduktion und Verbesserung der Qualität der Leistungen des Spitals und damit der Sicherheit des Patienten. Der wesentliche Ansatz besteht darin: nicht bei den wichtigen Folgen von Ereignissen anzusetzen, sondern bei den Ursachen, denn auch unwichtig scheinende Ursachen (unsichere Handlungen und Bedingungen) führen oft zu ernsthaften Folgen.

In Analogie zur Unfallpyramide kann man für die Situation medizinischer Komplikationen eine Komplikationenpyramide postulieren (Abb. 3).

Auch hier dürfte die Anzahl schwerwiegender Komplikationen proportional sein zur Anzahl unsicherer Handlungen und Bedingungen, wie man das anhand des Beispiels «Phlebitis» darstellen und nachweisen [3] kann. Durch Reduktion der Unsicherheiten und geringfügigen Komplikationen lassen sich die schwerwiegenden reduzieren. 
Abbildung 2

Die Unfallpyramide.

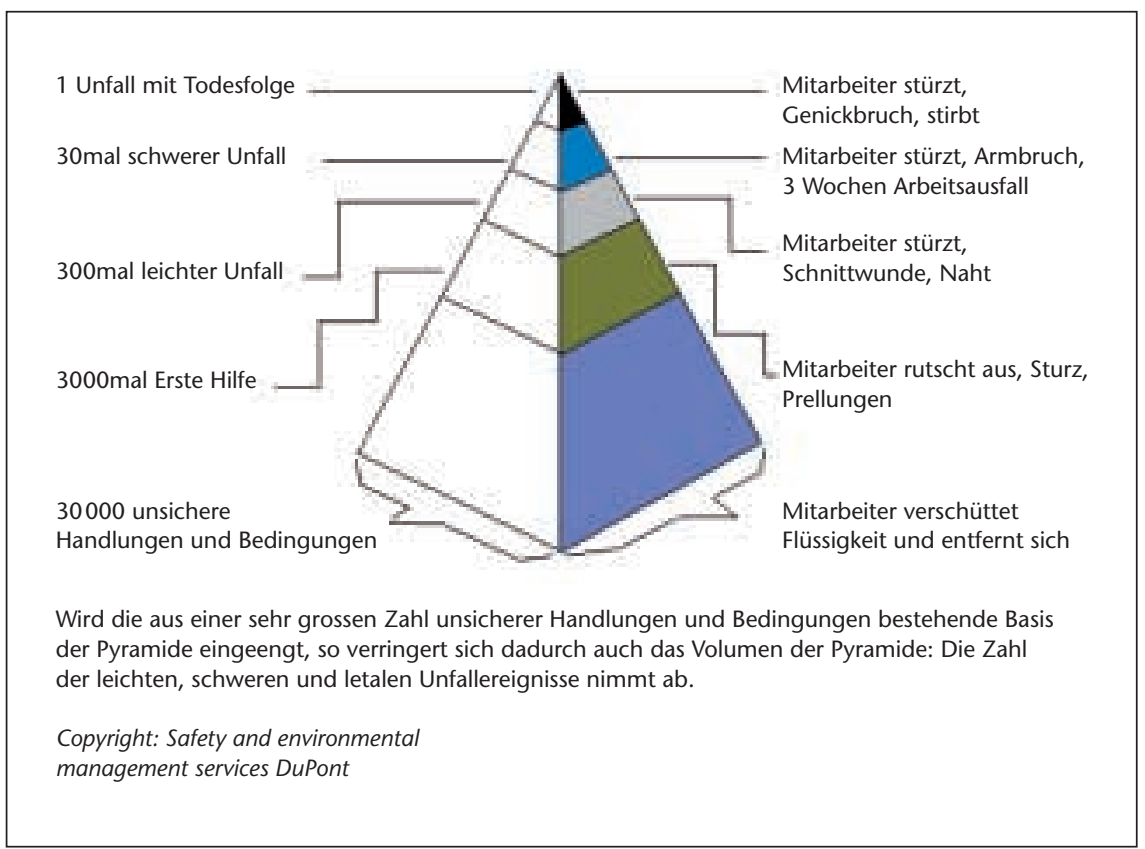

Abbildung 3

Die Komplikationenpyramide.

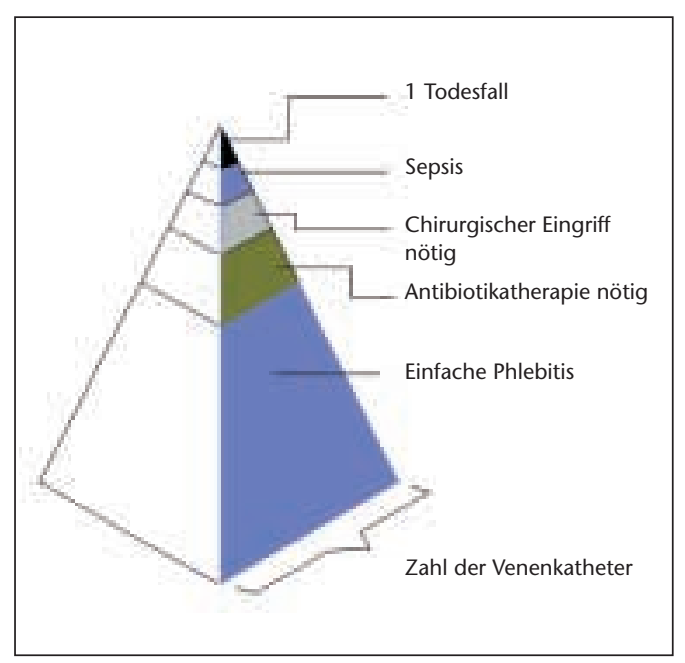

\section{Ziele}

Die Ziele des DuPont-Konzeptes für die Mitarbeiter in industriellen Betrieben lassen sich ana$\log$ für die in Spitälern behandelten Patienten wie folgt formulieren:

1. Erhöhung der Patientensicherheit;

2. Reduktion von Leiden, Leid und Kosten;

3. laufende Verbesserung der Qualität der Leistungen.
Ein wichtiger Aspekt bei der Umsetzung ist, dass die Verantwortung im Sicherheitsbereich nicht delegierbar ist: Sicherheit ist Chefsache. Voraussetzung für die sinnvolle Beteiligung am Programm sind folgende Punkte:

- absolutes Ernstnehmen der Sicherheit;

- Konsequenz in der Durchsetzung, weil schlechte Beispiele die Disziplin untergraben;

- der Ansatz für Verbesserungen besteht nicht in der Diskussion der Schuldfrage, sondern es sind die Gründe für das Abweichen von Standards zu klären und die nötigen Anpassungen zu machen.

\section{Praktisches Vorgehen}

In den beteiligten Kliniken für Innere Medizin wird über jeden stationären Patienten ein Rapportblatt erstellt (Multiple-choice-Tabelle), auf dem von 46 zur Evaluation vorgesehenen komplikationsträchtigen Interventionen die für den Patienten zutreffenden registriert werden sowie die dadurch allenfalls bedingten Komplikationen. Unter den evaluierten Interventionen figurieren sowohl ärztliche als auch solche der Behandlungspflege. Die Registrierungen erfolgen durch die Assistenzärztinnen/-ärzte, Kontrollen bzw. Ergänzungen durch Ober- und Chefärzte/ -ärztinnen. Das Datenblatt wird spätestens mit dem Spitalbericht fertiggestellt und in der zentralen Datenbank verarbeitet. Es ist klar, dass die Beteiligung eines Spitals an diesem Programm einen bescheidenen zusätzlichen Zeitaufwand pro Patient bedeutet, durchschnittlich 3-4 Minuten pro Spitalaufenthalt. Davon entfallen etwa $2 / 3$ auf den Assistenzarzt, $1 / 3$ auf das Kader.

\section{Auswertung und Verwendung der Resultate}

Von 1998 bis Ende September 2003 wurden die 5280 Komplikationen ausgewertet, die sich in einem beobachteten Kollektiv von $138000 \mathrm{~Pa}-$ tienten ereigneten. 26 Kliniken haben zu diesen Erhebungen beigetragen. Deren Resultate werden in jährlichen Statistikberichten zusammengefasst. In zweimal pro Jahr erscheinenden Bulletins (bisher 10 Nummern) werden die lehrreichsten Fälle (bisher 171) publiziert. Die Bulletins werden an alle medizinischen Kliniken und Abteilungen verschickt, auch an diejenigen, welche nicht an der Erhebung teilnehmen. Die Kliniken können anhand des kasuistischen Materials regelmässige Komplikationenkonferenzen durchführen und damit den Weiterbildungs- 
aspekt ausnützen, der sich aus diesem Programm ergibt. Darüber hinaus ergibt sich Handlungsbedarf in bezug auf die Anpassung oder die Erstellung von Richtlinien für die Abläufe im Spital. Solche Richtlinien festzulegen und vor allem sie durchzusetzen ist eine grosse, aber auch wirksame Herausforderung. Dazu eignet sich z. B. eine interdisziplinäre Konferenz für Patientensicherheit in jedem Spital, welche Sicherheitsthemen behandelt und die auch von Komplikationen in den andern Spitälern lernt, um sie im eigenen Spital zu vermeiden, auch wenn sie hier noch nie vorgekommen sind. In dieser Konferenz sollten Personen aus Ärzteschaft, Pflegedienst, Apotheke, Spitalhygiene, technischen Diensten und Administration permanent Einsitz nehmen sowie weitere Fachleute nach Bedarf beigezogen werden.

\section{Die Erfahrungen in der nun sechsjährigen Laufzeit des Programms führten zu folgenden Erkenntnissen und Vorschlägen zur Umsetzung der Resultate}

Bei verschiedensten Eingriffen

gibt es folgende kritische Punkte

- Identifikation von Patienten;

- Fehlen oder Nichtbeachtung von Richtlinien;

- Fehlen von Verbindlichkeiten/Durchsetzungsverfahren;

- Irrtümer auf verschiedensten Stufen in der medikamentösen Behandlung: Indikationsstellung, Verordnung (z. B. Schreibweise), Verabreichung, doppelte Buchführung;

- interdisziplinäre Zusammenarbeit.

Bei allen 5 Punkten spielen Probleme an den Schnittstellen die Hauptrolle.

\section{Vorschläge zur Umsetzung der Resultate}

\section{Schreibweisen}

- Änderung der Schreibweise der Insulineinheiten von IE auf E, um zu vermeiden, dass das I in der Bezeichnung IE als eine Ziffer 1 gelesen wird und deshalb eine zu hohe Insulinmenge gespritzt wird, also z. B. 41 Einheiten anstelle von 4 Einheiten. Wenn diese Änderung in einem Spital beschlossen wird, muss sie für alle Abteilungen am gleichen Tag in Kraft treten, da sonst zusätzliche Verwechslungsgefahr besteht.

- Schreibweise von Bruchstrichen bei handschriftlicher Verordnung: horizontal statt schräg, da dadurch geringere Verwechslungsgefahr von z. B. $1 / 2$ mit $1 \frac{1}{2}$ besteht.

\section{Handhabung von Medikamenten}

- Handhabung zurückgelassener patienteneigener Medikamente auf den Pflegestationen - Rückschub in die Apotheke.

- Präzisierungen für den Ablauf der Medikamentenverteilung an die Patienten auf den Spitalabteilungen. U.a. dürfen Medikamente nicht in unangeschriebenen Behältern ans Patientenbett gebracht werden.

- Richtlinien für die Behandlung von Patienten auf Transporten ausserhalb des Spitals, beispielsweise für Infusionen mit Opioiden oder Heparin.

- Änderungen im Medikamentensortiment: Wechsel auf eine einzige Dosierungsstärke bei den Haldol-Tropfen und bei Xylocain für die Lokalanästhesie ausserhalb des anästhesiologischen Bereichs.

- Medikamentenverordnungen durch Konsiliar- und Belegärzte: Abgrenzung der Verordnungskompetenzen.

\section{Prozedurale Richtlinien}

- Vorbereitung für Koloskopie: Vorschläge für die Wahl des Abführmittels und die Patientenkontrolle während der Vorbereitung.

- Bettflaschen: Verbot wegen immer wieder vorgekommener Verbrennungen.

- Telemetrieüberwachung: Vorschläge zur Verbesserung des Informationsflusses.

- Blasenkatheter: Klare Regelung zur Verordnungskompetenz gefordert.

- Seitenlokalisation: Richtlinien zur Vermeidung von Seitenverwechslungen, zum Beispiel bei Pleurapunktionen.

- Sauerstoffverordnung: Schriftliche Verordnung gefordert wegen vorgekommener $\mathrm{CO}_{2}$ Narkosen.

- Patientenidentifikation: Zur Vermeidung von Patientenverwechslungen bei Untersuchungen ausserhalb des Patientenzimmers wurden besondere Massnahmen vorgeschlagen.

- Invasive Eingriffe: Gerinnungsverhältnisse: Verwendung einer vorgeschlagenen Checkliste zur Evaluation der Blutungsneigung.

- Die Injektion von Heparinpräparaten zur Thromboseprophylaxe und -therapie muss sehr langsam erfolgen (z.B. kann man von der zu spritzenden Menge zunächst ein Drittel spritzen, dann 15 Sekunden warten, das zweite Drittel spritzen, wieder 15 Sekunden warten, dann das letzte Drittel spritzen. In dieser Art sollten auch die Patienten für Selbstinjektion instruiert werden [5]). Damit können die bei rascher Injektion oft auftretenden Hämatome weitestgehend vermieden werden. Injektionen in die Bauchdecke sollten unterlassen werden (siehe unten). 


\section{Schriftliche Dokumentation}

- Doppelte Buchführung: Abschaffung doppelter Buchführung, z. B. bei der Antikoagulation und der Diabeteskontrolle, damit keine Missverständnisse wegen Doppelspurigkeiten und asynchroner Dokumentation auftreten.

- Allergiepass: Ausfüllen am Anfang der Hospitalisation und laufende Ergänzung, wann immer eine neue Allergie auftritt, sowie gut sichtbare Aufbewahrung im Kardex, damit der Pass schon im Spital etwas nützt und nicht erst bei Spitalaustritt.

\section{Patienteninformation}

Patienteninformation über invasive Eingriffe: Diese kann aufgrund der hauseigenen und querverglichenen Erfahrungszahlen betreffend Komplikationen genauer erfolgen.

\section{Zahl der Eingriffe}

Änderung der Interventionshäufigkeit: Anhand des Programms kann die Interventionshäufigkeit für alle 46 evaluierten medizinischen Eingriffe in den Spitälern querverglichen werden. Eingriffe, die im Vergleich zu andern Kliniken häufiger durchgeführt werden, können kritisch hinterfragt und eventuell in ihrer Zahl reduziert werden. Beispiel: Durch Verminderung der Zahl der Eingriffe (z. B. Blasenkatheter) kann auch die Zahl der damit verbundenen Komplikationen verringert werden [1].

\section{Förderung der Weiterbildung}

Interdisziplinäre kasuistische Komplikationenkonferenz zur Besprechung der Komplikationsfälle des eigenen Spitals und der andern Spitäler als regelmässige Weiterbildungsveranstaltung.

\section{Umsetzung von Richtlinien}

Interdisziplinäre Konferenz für die Patientensicherheit zwecks Implementierung von Richtlinien, die sich aus Komplikationsfällen des eigenen Spitals ableiten lassen, sowie aus Fällen, die sich in den andern Spitälern ereignet haben.

\section{Beispiel für ein spezifisches und - wegen seiner Seltenheit - bezüglich Gefährlichkeit unterschätztes Problem}

\section{Das Bauchwandhämatom}

In den Jahren 1998 bis 2003 wurden Bauchwandhämatome bei 61 Patienten rapportiert. Die Patienten waren grösstenteils antikoaguliert, zum Teil überlappend parenteral und oral, zum Teil kombiniert mit Thrombozytenaggregations- hemmern. In $43 \%$ waren Erythrozytentransfusionen nötig. Tabelle 1 fasst weitere Informationen zusammen.

Fünf Patienten verstarben in kausalem $\mathrm{Zu}$ sammenhang mit der Blutung, entsprechend einer Letalität von $8 \%$ (Tab. 2).

Diese Fallsammlung lässt vermuten, dass folgende Umstände Bauchwandhämatome begünstigen:

1. Antikoagulation;

2. Injektion von Heparinpräparaten in die Bauchdecke;

3. Injektionen in die Bauchdecke beim antikoagulierten Patienten, auch wenn es sich bei den Injektionssubstanzen nicht um Antikoagulanzien handelt;

4. Husten als mechanisches Moment der Bauchdeckenbeanspruchung;

5. weibliches Geschlecht.

Die Literatur bestätigt, dass das Bauchwandhämatom eine relativ seltene Komplikation ist, dass die meisten Patienten mit dieser Komplikation antikoaguliert sind und dass Heparininjektionen in die Bauchdecke, Husten und weibliches Geschlecht das Auftreten begünstigen. Auch Narben sollen die Blutungen begünstigen [6]. Die Komplikation sollte möglichst konservativ behandelt werden [6-9]. Das Durchschnittsalter unserer Patienten liegt höher als in der zitierten Literatur. Die von uns beobachtete höhere Letalität hängt möglicherweise damit bzw. mit der entsprechenden Bindegewebsatrophie zusammen.

Bauchdeckenhämatome sind bei den älteren, oft polymorbiden internistischen Patienten prinzipiell gefährlich. Weder Ruhigstellung noch konservative mechanische Blutstillung durch Kompression der blutenden Region sind möglich. Diese Komplikation wird bezüglich Bedrohlichkeit für das Überleben wahrscheinlich unterschätzt. Sie bedarf strikter Überwachung und ausreichender Transfusionsbehandlung. Kontrolle auf einer Intensivstation ist eventuell angezeigt. Unterstützung der Atmung kann wegen der mechanischen Behinderung durch das Hämatom wichtig sein. Extrapoliert man aus unseren Zahlen (5 Todesfälle auf 138000 Patienten) die Häufigkeit des tödlichen Bauchwandhämatoms für den Bereich der Inneren Medizin in der Schweiz, so kommt man auf 11 Todesfälle pro Jahr für die rund 312000 internistisch hospitalisierten Patienten [4]. Die Innere Medizin beherbergt aber nur rund ein Viertel aller hospitalisierten Patienten in der Schweiz, so dass die Mortalität in unserem Land gesamthaft höher liegen dürfte. 
Tabelle 1

Bauchwandhämatome in einem Kollektiv von 138000 Patienten internistischer Abteilungen von 1998 bis 2003.

\begin{tabular}{|c|c|c|}
\hline & Zahl der Patienten & $\%$ \\
\hline Zahl der gemeldeten Fälle & 61 & 100 \\
\hline Alter & $75 \pm 11$ & \\
\hline Geschlecht & $21 \mathrm{~m}, 40 \mathrm{f}$ & $34 \mathrm{~m}, 66 \mathrm{f}$ \\
\hline \multicolumn{3}{|l|}{ Antikoagulation } \\
\hline oral & 27 & 44 \\
\hline unfraktioniertes Heparin & 18 & 30 \\
\hline niedermolekulares Heparin & 21 & 34 \\
\hline $\begin{array}{l}\text { Injektion therapeutischer Dosen von niedermolekularem Heparin } \\
\text { in die Bauchdecke }\end{array}$ & 7 & 12 \\
\hline Multiple Injektionen von Insulin in die Bauchdecke am gleichen Tag & 1 & 2 \\
\hline Aspirin & 13 & 21 \\
\hline Husten & 7 & 12 \\
\hline \multicolumn{3}{|l|}{ Therapie der Blutung } \\
\hline Ec-Transfusionen & 26 & 43 \\
\hline Frischplasma-Transfusionen & 8 & 13 \\
\hline Operation & 3 & 5 \\
\hline Punktion & 1 & 2 \\
\hline tödlicher Verlauf & 5 & 8 \\
\hline
\end{tabular}

51 PatientInnen waren antikoaguliert. 11 davon wurden gleichzeitig mit Aspirin behandelt. 2 weitere Patientlnnen waren nur mit Aspirin behandelt. 8 PatientInnen erhielten weder Antikoagulanzien in therapeutischer Dosis noch Thrombozytenaggregationshemmer.

Tabelle 2

Tödliche Bauchwandhämatome.

\begin{tabular}{|c|c|c|c|c|c|c|c|c|}
\hline $\begin{array}{l}\text { Alter } \\
\text { Geschlecht }\end{array}$ & Grundkrankheit & 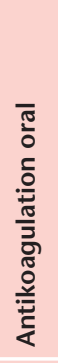 & 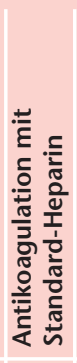 & 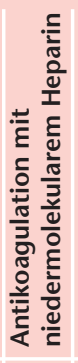 & & 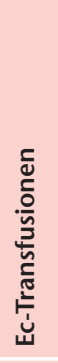 & 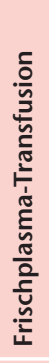 & Bemerkungen \\
\hline$>85 \mathrm{f}$ & Lungenembolie & & $x$ & & & 3 & & Quick 70\%, TZ I >60, II $15 \mathrm{sec}$ \\
\hline $59 \mathrm{~m}$ & $\begin{array}{l}\text { Hirnblutung, } \\
\text { Zystofixeinlage }\end{array}$ & & & & & $>2$ & 2 & $\begin{array}{l}\text { Verletzung eines arteriellen Bauch- } \\
\text { wandgefässes; nach initialer } \\
\text { Stabilisierung weiterer Hb-Abfall } \\
\text { ohne sicher erkennbare Blutung }\end{array}$ \\
\hline $83 \mathrm{~m}$ & $\begin{array}{l}\text { Glomerulonephritis, } \\
\text { nephrot. Syndrom, } \\
\text { Niereninsuffizienz, } \\
\text { Vorhofflimmern }\end{array}$ & & & $x$ & $x$ & 4 & 3 & $\begin{array}{l}\text { Fragmin } 15000 \mathrm{E} / \mathrm{d} \text {, Aspirin } 100 \mathrm{mg} / \mathrm{d} \text {, } \\
\text { Prednison } 5 \mathrm{mg} / \mathrm{d} \text { seit } 17 \text { Monaten, } \\
\text { Hämatom ausgehend von Injektions- } \\
\text { stelle in Bauchhaut, Körpergewicht } 98 \mathrm{~kg}\end{array}$ \\
\hline $86 f$ & $\begin{array}{l}\text { Niereninsuffizienz, lokal } \\
\text { begrenztes Mamma- } \\
\text { carcinom, beginnende } \\
\text { Demenz, Lungen- } \\
\text { embolie }\end{array}$ & $x$ & & & & 2 & 2 & $\begin{array}{l}\text { tiefster Quick } 31 \% \text {, INR } 2.07 \text {, Hb sank } \\
\text { von } 119 \text { auf } 74 \mathrm{~g} / \mathrm{l} \text {, starker Husten } \\
\text { trotz Codein, Kreatininclearance } \\
26 \mathrm{ml} / \mathrm{min}\end{array}$ \\
\hline $79 \mathrm{f}$ & $\begin{array}{l}\text { Polymorbidität } \\
\text { Lungenembolie }\end{array}$ & $x$ & $x$ & $x$ & & 2 & 2 & $\begin{array}{l}\text { Bauchwandhämatom mit Anurie, } \\
\text { foudroyant, INR 3.5, Prothromplex }\end{array}$ \\
\hline
\end{tabular}


Prophylaxe und Therapie sollten folgendes umfassen:

1. Injektionen von Antikoagulanzien in die Bauchdecke sollen wenn immer möglich unterlassen werden (Ausnahme in der frühen Schwangerschaft [5]);

2. auch Injektionen anderer Art in die Bauchwand sollten vermieden werden, wenn jemand antikoaguliert ist;

3. Patienten mit Bauchwandhämatom müssen sorgfältig überwacht werden und Transfusionsbehandlung grosszügig erhalten. Die Antikoagulation soll wenn möglich abgebrochen, mindestens aber für 12 bis 36 Stunden völlig aufgehoben werden. Bei unbedingt notwendiger Wiederaufnahme der Antikoagulation ist eine einschleichende Standardheparintherapie (z.B. Liquemin) niedermolekularem Heparin vorzuziehen, da eine Antagonisierungsmöglichkeit mit Protamin gegeben ist. Supportive Massnahmen wie Atemunterstützung sollten erwogen werden.

Das Beispiel des Bauchwandhämatoms zeigt den Nutzen klinik- und spitalübergreifender Anstrengungen für die mögliche Prophylaxe seltener Komplikationen im gesamtschweizerischen Rahmen. Die Häufigkeit von 11 tödlichen Ereignissen pro Jahr bedeutet 1 solche Komplikation etwa alle 16 Jahre pro medizinische Klinik, eine für die einzelne Klinik also kaum alarmierende Situation. Die wesentliche Lehre daraus ist aber, dass wir landesweit zur Senkung der durch diese Komplikation bedingten Morbidität und Mortalität und damit zur Erhöhung der Patientensicherheit durch die einfachen Prophylaxemassnahmen beitragen sollten, auch wenn diese Komplikation nicht zur persönlichen Erfahrung einer bestimmten Spitalbelegschaft gehört.

\section{Kommentar}

Das Programm hält die Komplikationen medizinischer Handlungen systematisch fest. Die Konfrontation und der institutionalisierte Umgang mit den Unzulänglichkeiten und Fehlern, den negativen Aspekten unserer Bemühungen um die Patienten, wird dadurch zu einer Selbstverständlichkeit und einer Quelle für Verbesserungen. Die Systematik des Vorgehens rechtfertigt sich auch dadurch, dass jeder Patient darauf Anspruch haben sollte, dass man sich die Frage stellt, was gut- und was schlechtgegangen sei, die entsprechende Lehre für die andern Patienten optimal nützt und sie auch andern Kollegen und medizinischen Fachleuten vermittelt. Die Komplikationenliste erfüllt diesen Zweck.
Indem sowohl Interventionen als auch Komplikationen bei jedem Patienten erfasst werden, ist die Möglichkeit gegeben, den prozentualen Anteil der Eingriffe einer bestimmten Art, die zu Komplikationen führen, anzugeben und damit die Patientenaufklärung vor einem Eingriff im eigenen Spital präziser zu gestalten. Umgekehrt weiss man auch, welcher Anteil der Interventionen komplikationsfrei verläuft.

Systematik der Erfassung und entsprechende Pflege der Details, auch im Bereich der Komplikationen, erzeugen in den entsprechenden Kliniken ein Klima der besonderen Sorgfalt im Umgang mit den Patienten. Das ist ein wichtiger, willkommener und erwarteter Effekt dieser patientenorientierten Arbeit.

Sie ist nur möglich unter der Voraussetzung, dass sich der Chef mit den Grundsätzen des Programms identifiziert und die Verantwortung für die Umsetzung der Erkenntnisse aus den Komplikationsereignissen im eigenen Spital und in andern Spitälern übernimmt. Wenn man zu diesem Zweck eine interdisziplinäre Konferenz für die Patientensicherheit einberuft, wird man feststellen, dass die Teilnehmer aus allen möglichen Fachbereichen kreative Beiträge liefern.

Der Klarheit halber sei hier angefügt, dass aus der statistischen Aufarbeitung und der Fallsammlung lediglich Empfehlungen für Massnahmen hervorgehen, jedoch keine bindenden spitalübergreifenden Richtlinien. Die daraus abgeleiteten Konsequenzen sind Sache der einzelnen Kliniken.

Die statistischen Aussagen dieses multizentrischen Programms zu den Zwischenfällen und den unsicheren Handlungen und Bedingungen unterliegen folgender Beschränkung: Trotz des dargestellten Grundsatzes, dass auch unsichere Handlungen und Bedingungen, die keine konkreten Folgen für den Patienten haben, erfasst werden sollen, müssen wir davon ausgehen, dass solche Ereignisse nicht immer registriert werden und sich die Dokumentation oft eher auf Ereignisse mit Auswirkungen bei den Patienten konzentriert. Auch gibt es einen Ermessensspielraum z. B. zur Frage: Wo beginnt die unsichere Handlung bzw. Bedingung? Die statistischen Angaben zu Komplikationsereignissen mit Folgen für die Patienten sind deshalb wahrscheinlich zuverlässiger als zu solchen ohne klinische Folgen. Häufigkeitsangaben zu allen unerwünschten Ereignissen, die sich auf eine spitalübergreifende Grundgesamtheit von beobachteten Patienten beziehen, müssen deshalb prinzipiell als Minima betrachtet werden. 
Literatur

1 Stäubli M. Komplikationen medizinischer Interventionen. Komplikationenliste der Chefärztevereinigung der Schweizerischen Gesellschaft für Innere Medizin. Schweiz Ärztezeitung 2001; 82:1331-8.

2 Stäubli M. Komplikationen in der Inneren Medizin. Z Arztl Fortbild Qualitatssich 2001;95:485-8.

3 Stäubli M, Suter J. Beitrag zur Qualitätssicherung in der Inneren Medizin. In: Cranovsky R, Hurter $\mathrm{H}$. Risikomanagement und Patientensicherheit in der Behandlung. Aarau: Verlag H+Bildungszentrum; 2003. S. 71-82.

4 Bundesamt für Statistik. Spitalstatistik 2002.

5 von Felten A. Thrombose- und Hämostase-Labor (Diagnostica), Dufourstrasse 90, Zürich. Persönliche Mitteilung.

6 Verhagen HJ, Tolenaar PL, Sybrandy R. Haematoma of the rectus abdominis muscle. Eur J Surg $1993 ; 159: 335-8$

7 Lohri A, Petralli C, Kummer H. Bauchwandhämatome als schwere Komplikation der Antikoagulation. Klinik und Ultraschalldiagnose. Schweiz Med Wochenschr 1984;114:1761-2.

8 Egger B, Schweizer W, Wagner HE. Das akute Abdomen bei Bauchwandblutung unter Antikoagulation. Helv Chir Acta 1992;59:399-402.

9 Blum A, Bui P, Boccaccini H, Bresler L, Claudon $\mathrm{M}$, Boisset $\mathrm{P}$, Regent D. Imagerie des formes graves de l'hématome des grands-droits sous anticoagulants. J Radiol 1995;76:267-73.

\section{Am Programm beteiligte Personen und medizinische Kliniken der Spitäler}

J. P. Bernhardt, Hôpital de Porrentruy; P. Bischof, M. Pfister, Spital Rorschach; B. Caduff, B. Nyfeler, S. Buchholz, M. Schwanda, Spital Limmattal; Y. Crippa, C. Beutler, Kantonales Spital Grabs; R. Fleisch, Klinik Schloss Mammern; U. Gössi, P. Eichhorn, Spital Schwyz; P. J. Hangartner, R. Blank, M. Rhyner, Spital Altstätten; F. Kocher, J. Brühwiler, A. Spillmann, Bezirksspital für das Freiamt, Muri; U. Marbet, Kantonsspital Uri, Altdorf; U. Münch, A. Ciurea, Spital Bülach; M. Negri, S. Moser, Spital Frutigen; M. Pirovino, C. Züger, Kantonsspital Olten; F. Repond, U. Baumann, R. Grunder, Regionales Spitalzentrum Münsingen; P. Risi, Bezirksspital Erlenbach im Simmental; S. Rüttimann, Kantonsspital Schaffhausen; P. Schlup, A. Oestmann, B. Qarolli, Regionalspital Grenchen; M. Schmidli, Kantonales Spital Herisau; D. Schmidt, Kantonales Spital Walenstadt; R. Schoenenberger, Bürgerspital Solothurn; M. Spiegel, Spital Uster; M. Stäubli, U. Senn, J. Burkhard, Spital Zollikerberg; M. Vogt, Zuger Kantonsspital; H. Vontobel, B. Walther, Spital Wetzikon; A. Walser, W. Koch, Spital Flawil; D. Wegmann, Kantonsspital Nidwalden, Stans; U. Zellweger, Spital Ilanz.

\section{Dieses Programm wird ermöglicht dank der finanziellen Unterstützung durch folgende Institutionen}

Pharmazeutische Firmen ALTANA, GlaxoSmithKline, Bristol-Myers Squibb, UPSA, Sanofi-Synthélabo, Pfizer, MSD, Roche Pharma (Schweiz), Schweizerische Gesellschaft für Innere Medizin, Spitäler und private Donatoren. 